ISAHP 2001, Berne, Switzerland, August 2-4, 2001

\title{
A CHOICE OF A PERSPECTIVE SYSTEM FOR VIBRATION ISOLATION IN CONDITIONS OF VARYING ENVIRONMENT ${ }^{1}$
}

\author{
Alexander Andreichicov and Olga Andreichicova \\ Volgograd State Technical University, Lenin avenue 28, 400066 Volgograd Russia \\ andr@vstu.ru
}

Keywords: Analytic network process, external designing, limiting priority, supermatrix, dynamic judgments.

Summary: The report consider the application of AHP and ANP for prediction of probable modifications of the factors, which mainly influence a choice of a type of vibration isolation system for the manufacturing. Similar tasks take place in the problems of external designing, when it is necessary to make decision being effective in varying environment. ANP technique gives limiting impact priorities for influential factors when the experts' preferences are constant. AHP technique with use of the dynamic judgments enables to find out the tendencies of priorities changes based on the dynamic experts judgments, which are given by defined functions of time. The obtained outcomes are consistent and supplement each other.

\section{Introduction}

The decision making in the external designing influences a solution's choice during an internal designing of the engineering. The strategic solutions accepted during an external designing are founded on prediction of probable modifications of the object being designed and its environment taking into account an interaction between them. ANP gives good means for a solution of the similar tasks. If the elements of a system containing the designing object influence each other, their priorities permanently vary. The ANP (Saaty, 1996) enables to find out the tendencies of a priorities modification for system units concerning the given purpose and to determine the limiting values of these priorities. The limiting priorities can be interpreted as priorities of the system units in the future, i.e. as the probable prognosis for a case, when the preferences expressed will remain constant during forecasting period. Other approach to the forecasting offered by T.L. Saaty (Saaty, 1994), is based on dynamic expert judgments. The considered problem is represented by a hierarchy, and the expert preferences are set as the function of time. The handling of dynamic judgments enables to find out the dependencies of the task units' priorities in time.

It seems interesting to compare outcomes for different approaches during a solution of a concrete task in the external designing. The ANP approach takes into account mutual influences of the task units and enables to carry out the prognosis at the constant expert preferences. In AHP technique some relations between units of the task is not taken into account, however, the preferences may vary in time. In these techniques a structure of the task differs, however, a set of alternatives being compared is identical. Thus both ANP, and AHP enables to find alternatives, which will have the greatest impact into the formulated purpose in the future. The comparison of the obtained outcomes gives an opportunity to reveal contradictions in input data and to produce their sequential improvement.

${ }^{1}$ Acknowledgements. We thank Thomas L. Saaty, Rozann Saaty-Whitaker and Creative Decisions Foundation for supporting of our research. 


\section{Application of ANP technique for determination of the best vibroisolation system}

This report considers the task of the choice of a vibration isolation system (VIS) for vehicles, which will be perspective for manufacturing in Russia in the coming decade. The major factors and actors for a considered problem are represented in a figure 1, where arrows specify influence directions. This task includes 29 elements, which are joined in eight clusters. The first cluster contains the alternative VIS variants offered for production. Among them there is a coil spring, which frequently is used now in vehicle suspensions. The coil springs are simple for manufacturing, they have high reliability, low cost and good operational properties. VIS of this type have not a very high vibroisolation quality, which, besides depends on weight of the vehicle. At configuration of the vehicle a coil spring require significant space for layout. All these items restrict an application of such VIS type.

Pneumatic VIS have a very good vibroisolation quality, provide automatic tuning on different masses of the vehicle, can damp vibrations in various planes, have small sizes and have not a very high cost. Usage of air suspensions requires an energy supply and an protecting from mechanical damages. These requirements not always can be satisfied by the vehicle producers. VIS controlled enable to provide the excellent vibroisolation quality, an automatic tuning on a wide spectrum of vibrations, an improving a controllability of the vehicle. They have small sizes and good operational properties. The limiting factor for their usage now are the requirement of an energy supply, complexity of a manufacturing and high cost.

The properties of VIS being selected for manufacture influences the vehicle buyers requirements connected with vibration isolation (cluster 8). It also influences the VIS producers (cluster 2). The type of VIS influences the cluster 2 more than the cluster 8 (priorities vector $w=\{0.75 ; 0.25\}$ ).

The second cluster contains the purposes of the VIS producers (profit, manufacture growth, competitiveness of producible systems, engaging of the investments). The purposes of the VIS producers influence the type of VIS (1), the technologies being applied (4), the investors (6), the macroeconomic factors (5) and R\&D innovations (7). Besides, the purposes of the producers influence each other. The pair comparison matrix for these factors demonstrates, what of them is more influenced by the producers.

$$
\begin{array}{l||llllllc||}
\mathbf{1} & \mathbf{1} & \mathbf{2} & \mathbf{4} & \mathbf{5} & \mathbf{6} & \mathbf{7} & \boldsymbol{w} \\
\mathbf{2} & 1 & 1 & 3 & 3 & 5 & 6 & 0.316 \\
\mathbf{4} & & 1 & 3 & 3 & 5 & 6 & 0.316 \\
\mathbf{5} & & & 1 & 1 & 3 & 5 & 0.135 \\
\mathbf{6} & & & & 1 & 3 & 5 & 0.135 \\
\mathbf{7} & & & & & 1 & 3 & 0.062 \\
& & & & & & 1 & 0.035
\end{array}
$$

The principal requirements of the vehicle producers to the VIS are concentrated in the third cluster. These requirements include a high quality of vibroisolation, a high reliability, good layout properties of VIS (small dimensions, possibility of various orientation at the setting in the vehicle etc.) and an acceptable cost. The requirements of the vehicle producers influence the type of VIS (1), the possible investors (6) and R\&D innovations (7). Influence of the vehicle producers on the VIS type is stronger than ones on the clusters 6 and 7 (priorities vector $w=\{0.6 ; 0.2 ; 0.2\}$ ).

The fourth cluster contains criteria for an estimation of the technologies which might be used for VIS manufacturing, such as an availability, prospects, cost, power consumption, availability of materials and qualification of the staff. Availability of a technology, first of all, is meant as its existence and presence in the market. A technology cost also can make its unavailable to the producer, however, it is the other criterion. A 
technology can be present at the market and have the acceptable price, but to require a use of poorly accessible materials or too high qualification of a staff. For an estimation of these factors the criteria Materials required and Qualification required are used. The criteria for an technologies estimation are important only for the VIS producers and do not influence other clusters of the task, therefore in this case a pair comparison matrix shouldn't fill.

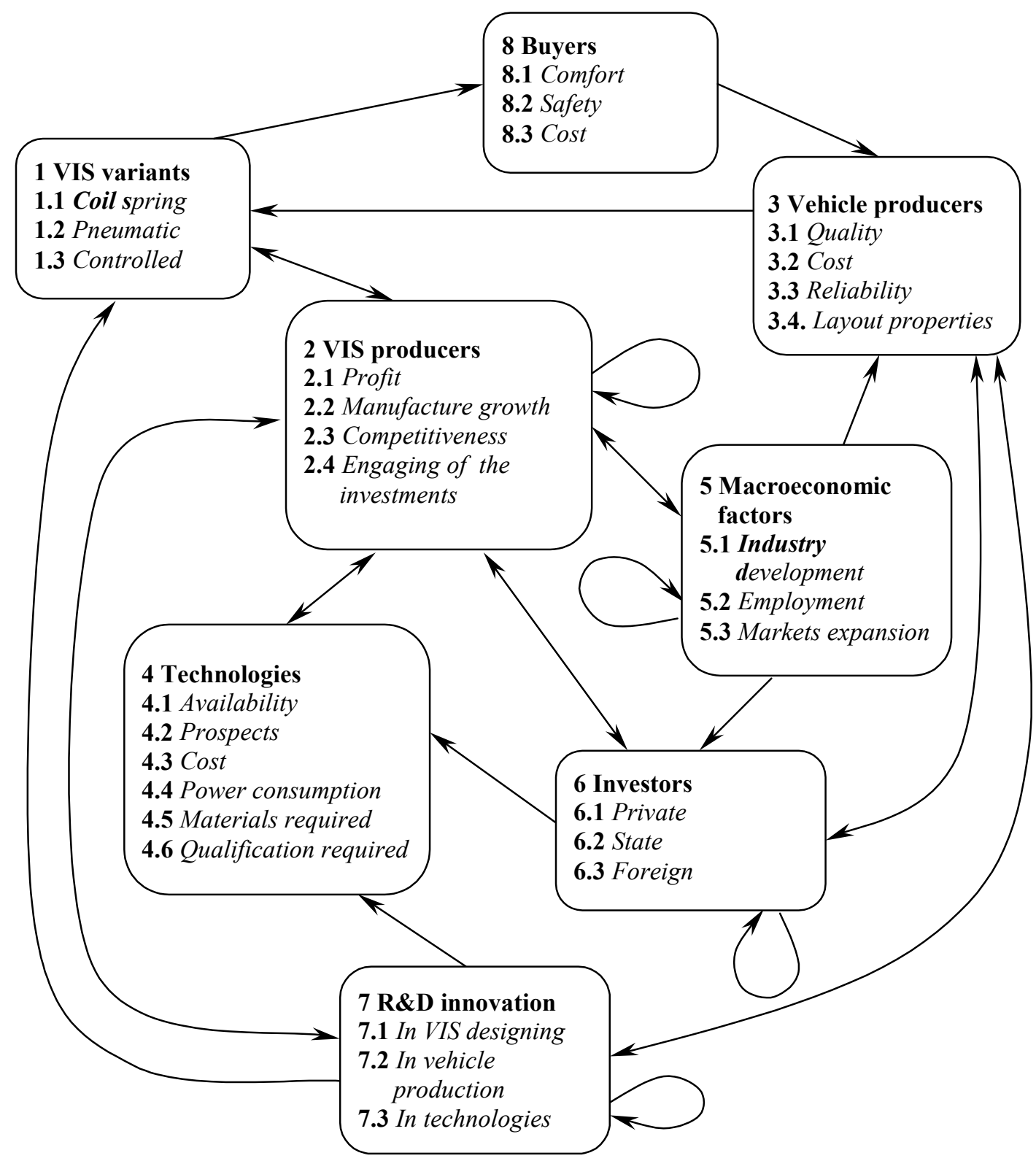

Figure 1. The network structure of the task of forecasting a perspective VIS 
Macroeconomic factors influence the VIS producers (2) and vehicle producers (3), and also the investors (6). Besides, they influence each other (5). The pair comparison matrix, indicating influence of the macroeconomic factors, looks like:

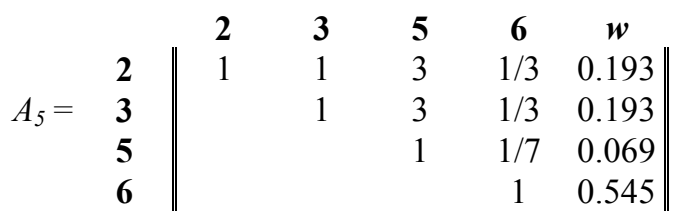

The types of the possible investors are included in a cluster 6 . The investor's interests essentially differ. The investors can influence the VIS producers (2), the vehicle producers (3), the technologies choice (4) and each other (6). The influence of the investors on the technology choice means that is not indifferent for them, what a technology to invest.

$$
A_{6}=\begin{array}{c||ccccc} 
& \mathbf{2} & \mathbf{3} & \mathbf{4} & \mathbf{6} & \boldsymbol{w} \\
\mathbf{2} & 1 & 1 / 5 & 1 / 3 & 3 & 0.116 \\
\mathbf{3} & & 1 & 3 & 8 & 0.573 \\
\mathbf{4} & & & 1 & 5 & 0.259 \\
\mathbf{6} & & & & 1 & 0.052
\end{array}
$$

The seventh information cluster of the task includes main directions of R\&D innovation, which influence the prospects of the VIS production. They are advanced designs in the field of VIS constructions, in the field of the technologies for VIS manufacturing and in the field of the new vehicles. The R\&D factors influence the following task units: the VIS producers (2), the vehicle producers (3), the technologies (4), and each other (7).

$$
A_{7}=\begin{array}{c||cccccc} 
& \mathbf{1} & \mathbf{2} & \mathbf{3} & \mathbf{4} & \mathbf{7} & \boldsymbol{w} \\
\mathbf{2} & 1 & 3 & 1 & 1 & 3 & 0.273 \\
\mathbf{3} & & 1 & 1 / 3 & 1 / 3 & 1 & 0.091 \\
\mathbf{4} & & & 1 & 1 & 3 & 0.273 \\
\mathbf{7} & & & & 1 & 3 & 0.273 \\
& & & & & 1 & 0.091 \|
\end{array}
$$

The eighth cluster contains the buyers requirements to the vehicles' properties, connected with a type of VIS used. They are a comfort, a safety and a cost. The buyers requirements influence only the vehicles producers, therefore a pair comparison matrix shouldn't fill here.

The clusters priorities calculated are used as weight factors in the procedure of a supermatrix's reduction to the stochastic form. The stochastic supermatrix is shown in table 1. For its forming it was necessary to fill in 90 matrixes of pair comparison for the clusters elements, subject to influence of all other units of the influencing clusters. All matrixes were consistent $(C R<0.1)$. The sequential raising of the supermatrix in integer degrees has resulted to the vector of limiting impact priorities $w^{\infty}$, (showed in table 2), which remained constant during further magnification of a degree. 
The outcomes obtained permit to consider the VIS controlled as the most perspective for manufacturing in the future. The pneumatic VIS is at the second place, and the coil spring - at the latter. A difference of a leading system from others is rather appreciable. Therefore, if the preferences expressed by the experts will not essentially vary during the forecasting period, then the best for manufacturing will be the controlled VIS at existing relations between the task units.

The clusters' influence on the main goal is shown in a figure 2. The VIS choice is essentially influenced by the purposes of VIS producers, especially by the competitiveness. The type of VIS follows it. At the third place 
Table 1. Supermatrix for the task of a choice of the best long-term VIS

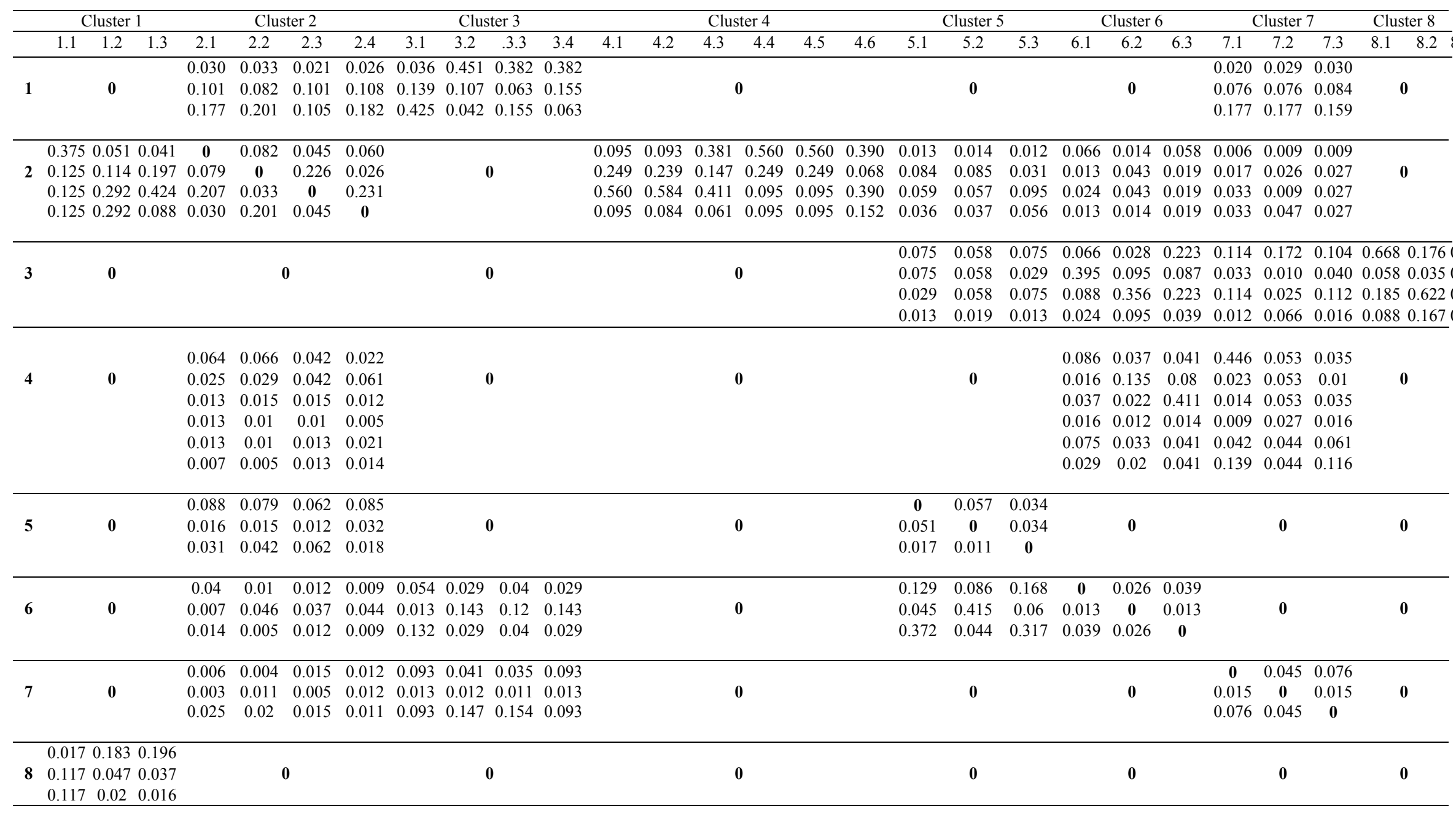


there are requirements of the vehicle producers to VIS. Vibroisolation quality and reliability are principal among them. Technologies parameters and investors influence a choice of VIS about equally. The most important technologies factors are availability and prospects. The influence of the state and foreign investors is stronger than of private ones. Influence of macroeconomic factors is not high in comparison with influence of other clusters. It may be explained by the psychology of experts, which are not inclined to the disastrous or too optimistic prognoses. The most important requirement of the vehicles' buyers to VIS is the comfort. The factors of R\&D innovations have a weak influence on the main goal in comparison with other units of the task. The major R\&D direction is a progress in the technologies.

Table 2. Limiting impact priorities

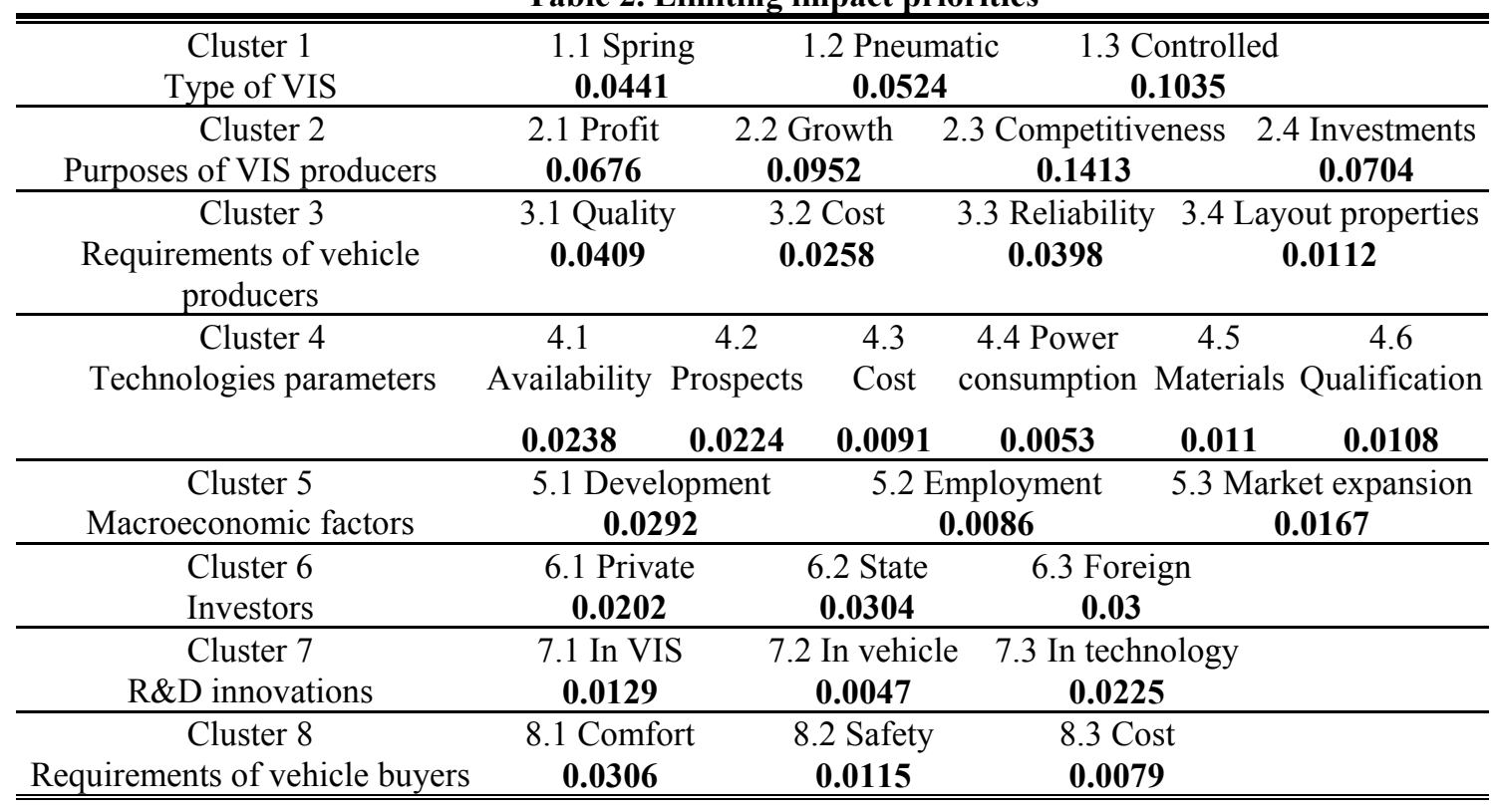




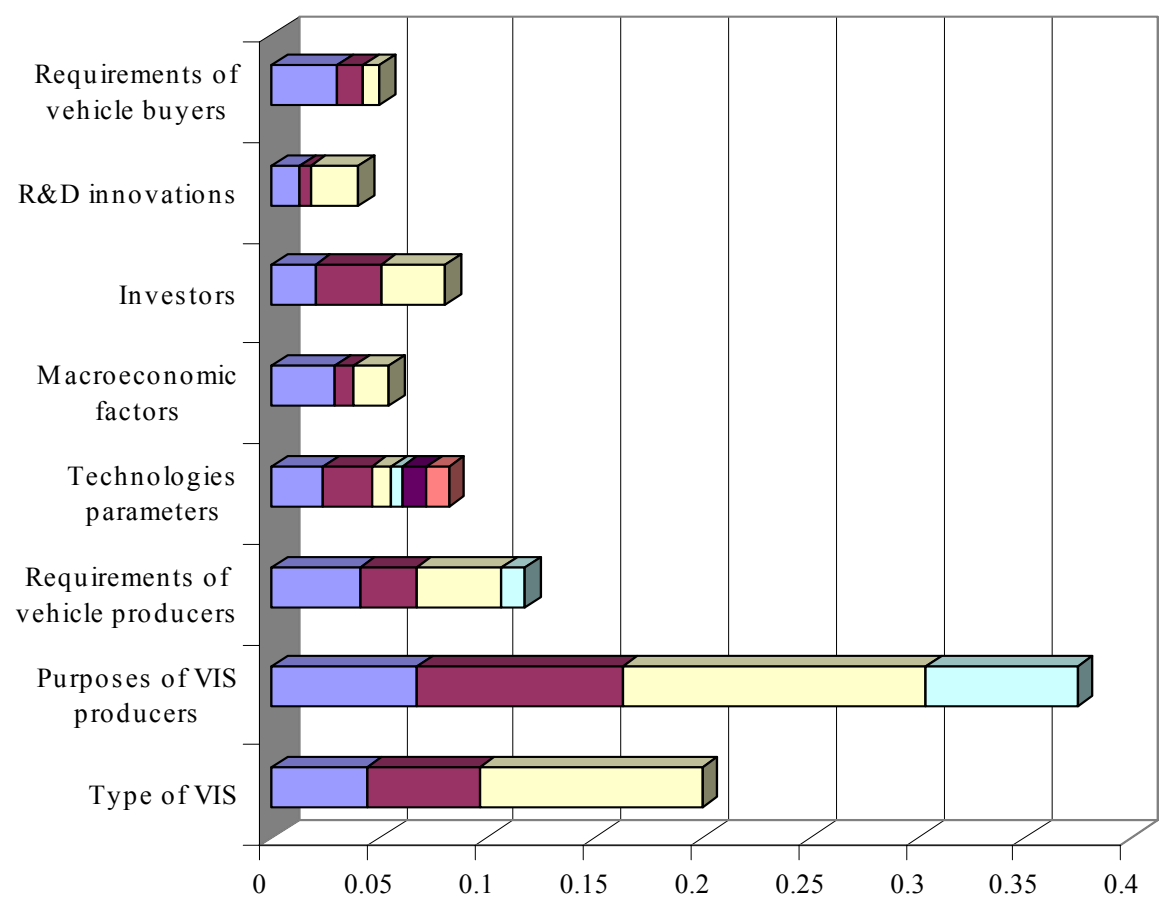

Figure 2. Limiting impact priorities for the clusters

The results obtained are in agreement with intuitive representations of the experts, are easily interpreted and give an opportunity to make a detailed prognosis substantiation. The intermediate results also are of interest, as enable to analyze probable interactions between units in a considered system. In comparison with traditional models of dynamic systems in form of the differential equations systems the considered approach has a number of advantages, such as the usage of the qualitative information and the time saving.

\section{The forecasting based on AHP with use of dynamic judgments}

We solved the task of a choice of VIS being perspective in the future with use of hierarchic approach. The task structure is shown in figure 3. A focus of the problem is a choice of a VIS type which will be the best in the future. The first level of hierarchy contains major factors influencing the actors located at the second level. Actors are the vehicles producers, VIS producers and technologies for VIS manufacturing. Actors are influenced by the macroeconomic factors, by $R \& D$ innovations, by an investment climate and by the buyers requirements. Every actor has the defined requirements to alternative types of VIS. Requirements sufficing is estimated by appropriate criteria. The criteria for an estimation of alternatives are located at the third level. The VIS producers are connected to the following criteria: profit; production growth; existence of the markets for selling; competitiveness. For the vehicles producers major criteria are vibroisolation quality, VIS reliability, cost and layout properties. The technologies for VIS manufacturing are connected with the criteria, such as a cost, a competitiveness and a simplicity of manufacturing. At the last, fourth level of hierarchy there are alternatives. They are a coil spring, a pneumatic VIS and VIS controlled. 


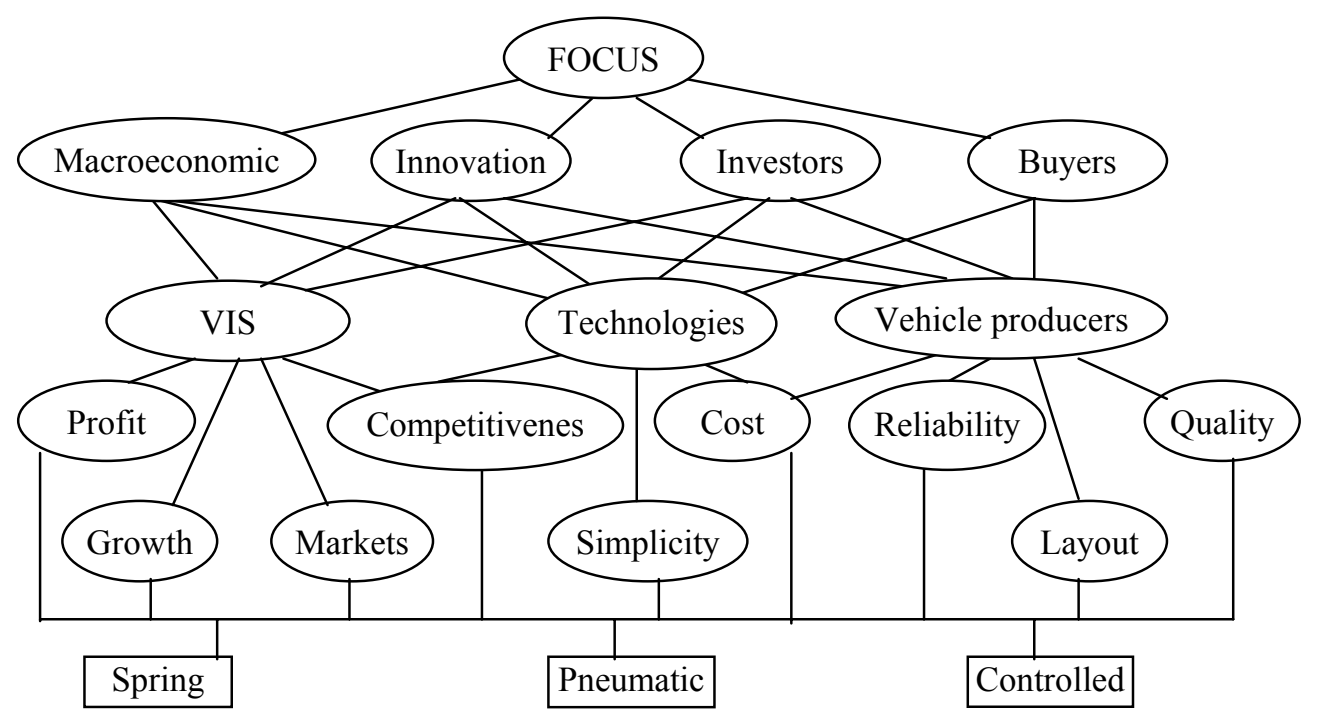

Figure 3. Hierarchy for the dynamic task

At first the static task for a choice of the best alternative was solved at the present moment $t_{0}$. All the pair comparison matrixes were consistent. The outcomes of the solution are represented by the set of priorities vectors shown in the table 3 . Then the varying preferences were determined. The most difficult thing in the expert forecasting consists in the definition of a concrete form for the tendencies of a modification of the defined parameters and in the accounting of their interaction. We assumed the preferences of considered alternatives concerning criteria of the third hierarchy level (table 4) will remain constants in the future. The preferences for the factors located at the first hierarchy level will vary.

Table 3. Priorities vectors for criteria in the static task

\begin{tabular}{lllll}
\hline \hline & Macroeconomic & Innovations & Investments & Buyers \\
FOCUS & 0.380 & 0.085 & 0.466 & 0.069 \\
\hline & VIS producers & Vehicles producers & Technologies & \\
Macroeconomic & 0.109 & 0.582 & 0.309 & \\
Innovations & 0.105 & 0.258 & 0.637 & \\
Investments & 0.167 & 0.740 & 0.094 & \\
Buyers & 0.167 & 0.833 & & \\
\hline & Profit & Growth & Competitiveness & Markets \\
VIS producers & 0.532 & 0.097 & 0.186 & 0.186 \\
\hline & Quality & Cost & Reliability & Layout \\
Vehicles producers & 0.143 & 0.402 & 0.054 & 0.402 \\
\hline & Competitiveness & Cost & Simplicity & \\
Technologies & 0.333 & 0.333 & 0.333 & \\
\hline \hline
\end{tabular}

The dynamic pair comparison matrixes were the followings:

\begin{tabular}{r|cccc||} 
FOCUS & Macroeconomic & Innovations & Investments & Buyers \\
Macroeconomic & 1 & $1 / 0.25 e^{0.6 t}$ & Auto & $1 /\left(0.2-0.05 t+0.08 t^{2}\right)$ \\
Innovations & $0.25 e^{0.6 t}$ & 1 & $0.2-0.18 t+0.15 t^{2}$ & Auto \\
Investments & auto & $1 /\left(0.2-0.18 t+0.15 t^{2}\right)$ & 1 & Auto
\end{tabular}




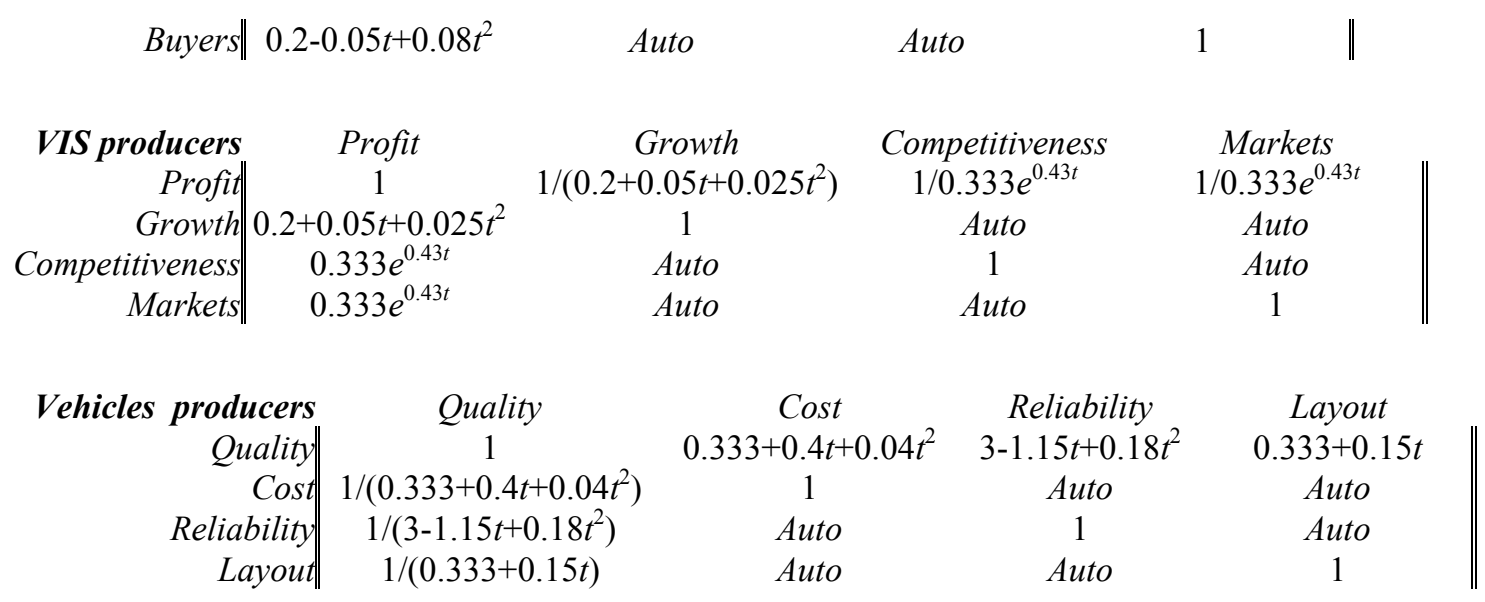

Table 4. Priorities vectors for alternatives in the static task

\begin{tabular}{llll}
\hline \hline & Coil spring & Pneumatic & Controlled \\
\hline Profit & 0.648 & 0.230 & 0.122 \\
Growth & 0.143 & 0.429 & 0.429 \\
Competitiveness & 0.075 & 0.333 & 0.592 \\
Markets & 0.109 & 0.582 & 0.309 \\
Quality & 0.066 & 0.319 & 0.615 \\
Cost & 0.751 & 0.178 & 0.070 \\
Reliability & 0.637 & 0.105 & 0.258 \\
Layout & 0.105 & 0.258 & 0.637 \\
Simplicity & 0.751 & 0.178 & 0.070 \\
\hline \hline
\end{tabular}

The relative importance of the criteria for each actor also will be subject to modifications in the future. The filling of pair comparison matrixes in the dynamic task was fulfilled as follows. There were selected $(n-1)$ cells in a matrix, where the functions describing a changes of appropriate preferences were formed. The preferences at the moment $t_{0}$ coincided with preferences in the static task. At the next moments the values for other $\left(n^{2}-2 n+1\right)$ preferences were calculated at the base of the $(n-1)$ functions given (Auto). Thus there was no problem of inconsistency during a solution of the dynamic task. The forming of functions was produced experimentally with the help of the developed software (Andreichicov and Andreichicova, 1999). Other pair comparison matrixes were the same as in the static task. The results are shown in the figures 4,5. 


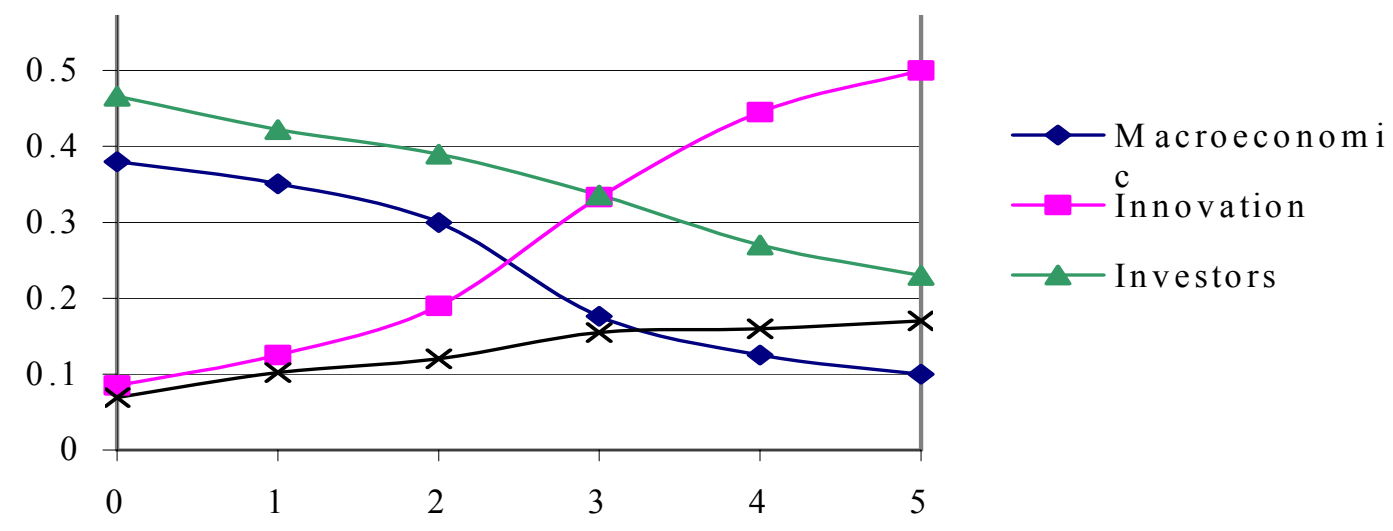

Figure 4. The changing of factor's priorities in time

Figure 5. The changes of alternative's priorities in time

The priority of innovations in Russia is supposed to increase in future. The buyers priority slightly will increase too. Investors importance and macroeconomic influence will decrease. These modifications and the

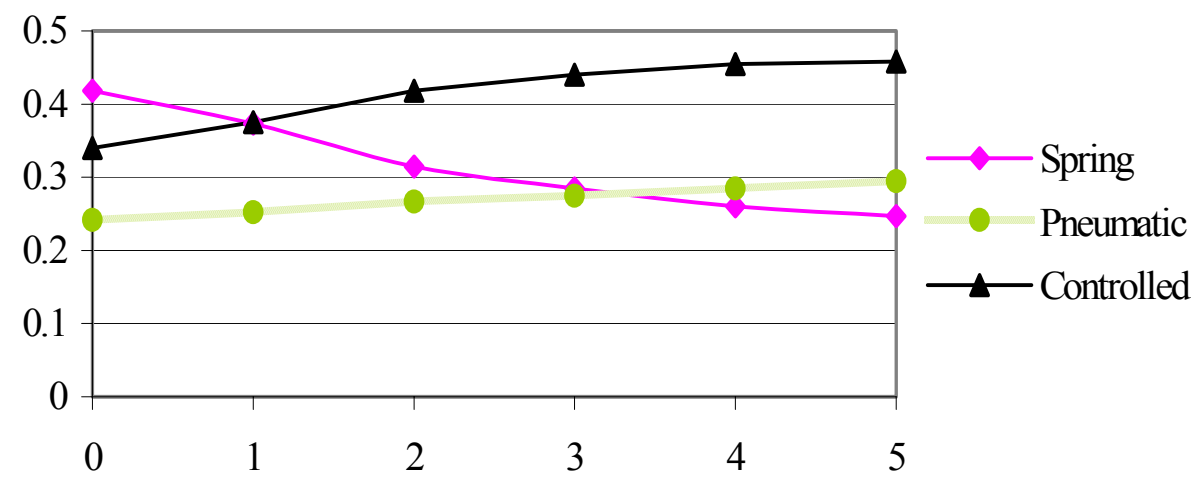

changes of criteria importance for producers will result in a changing of the order in the alternatives set. Spring coil, which is simple and cheap, moves from the first place to the latter. The best in the future will be VIS controlled, which has high vibroisolation quality and high cost. Low priority of the pneumatic VIS in both cases might be explained by its relatively high cost and low reliability.

\section{Discussion}

Both considered prediction methods show, that VIS controlled will be the most perspective system in the future. ANP gives a such outcome on the basis of an calculation of the limiting impact priorities for the task elements. The major factors in the choice are the VIS producers and their purposes. The most essential purpose is the competitiveness of producible designs. The interesting outcome is the correlation between limiting priorities of quality criteria being formulated by the vehicles producers. The most influential is the vibroisolation quality followed by reliability, cost and layout properties sequentially. The relation of these criteria priorities for the vehicles producers during a solution of the static task essentially differs from considered one. Major criteria for today are cost and layout properties, they are followed by quality and reliability. Solving the task on the base of AHP, we tried to use the most similar task units. It is necessary to 
notice these two approaches result in various statements of the task, which have the identical main goals, but different information sets and different models of interaction between information units. The solution of the dynamic tasks can be compared to a simulation modeling, which is fulfilled on the basis of a set of the suppositions formulated. Thus it is possible to realize a check of the various hypotheses of a probable preferences changes in the future.

\section{References}

1. Saaty T.L. (1996) Decision Making with Dependence and Feedback: The Analytic Network process, RWS Publications, Pittsburgh.

2. Saaty T.L. (1994) Fundamentals of Decision Making and priority Theory with the Analytic Hierarchy Process, RWS Publications, Pittsburgh.

3. Andreichicov A.V. and Andreichicova O.N. (1999) "Intelligent software based on AHP", Proceedings of the $5^{\text {th }}$ International Symposium on the Analytic Hierarchy Process, Kobe, 393-398. 